\title{
Descrição de um modelo prático para o aprendizado do acesso venoso periférico por estudantes de medicina e enfermagem
}

\author{
Description of a practical model for learning of peripheral venous access by students \\ of medicine and nursing
}

\section{Pedro Caetano Edler Zandoná ${ }^{1}$, Felipe Ferreira Laranjeira ${ }^{1}$, Eduardo Madalosso Zanin ${ }^{1}$, Luiza Machado Kobe ${ }^{1}$, Bruno Grund Frota ${ }^{1}$, Renata Silva Azevedo ${ }^{1}$, Daniel Weiss Vilhordo ${ }^{2}$, Ricardo Breigeiron ${ }^{3}$}

\footnotetext{
Acadêmicos da Faculdade de Medicina da Pontifícia Universidade Católica do Rio Grande do Sul (PUCRS). Membros da Liga do Trauma da PUCRS. Porto Alegre, RS.

2 Mestre em Medicina/Ciências Cirúrgicas. Cirurgião Geral e do Aparelho Digestivo do Hospital São Lucas da PUCRS. Orientador da Liga do Trauma da PUCRS. Porto Alegre, RS.

${ }_{3}^{3}$ Mestre em Medicina/Ciências Cirúrgicas. Professor Assistente da Faculdade de Medicina da PUCRS. Coordenador da Liga do Trauma da PUCRS.

Coordenador da Residência em Cirurgia Geral e do Trauma do Hospital de Pronto Socorro de Porto Alegre. Porto Alegre, RS.
}

\begin{abstract}
RESUMO
Objetivos: Descrever um modelo didático de acesso venoso periférico, simples e de baixo custo, desenvolvido pela Liga do Trauma da Pontifícia Universidade Católica do Rio Grande do Sul, que visa à aquisição de habilidades pelo estudante/profissional da saúde.

Métodos: Para reproduzir a via periférica de acesso utilizamos um garrote de borracha, simulando uma veia, com uma extremidade em fundo cego, inserida em uma luva de látex preenchida com estopa de algodão. A outra extremidade foi conectada a uma bolsa de solução parenteral contendo $500 \mathrm{ml}$ de soro fisiológico acrescido de corante, para simular sangue, através de equipos de infusão. A bolsa foi instalada em um suporte mais alto que a luva, facilitando a ação da gravidade. Com o regulador de fluxo completamente aberto, cria-se uma pressão através da presença do ar com o líquido dentro dos equipos de infusão e do garrote. A primeira punção com o cateter serve para retirar o ar de dentro das cânulas, o que permite a realização do procedimento.

Resultados: A punção, realizada de forma adequada, ocasiona o refluxo do sangue artificial por dentro do cateter, ao contrário do que acontece quando há transfixação do garrote de borracha. Isso simula uma situação real de punção venosa, pois a pressão hidrostática no garrote é análoga à encontrada em uma veia.

Conclusões: A descrição do modelo permite sua reprodutibilidade de maneira fácil, já que o mesmo utiliza materiais baratos e de fácil acesso. O modelo propicia a familiarização inicial com o procedimento, facilitando a prática no paciente ou em modelos mais sofisticados, ainda indispensáveis para o aprimoramento da técnica.
\end{abstract}

DESCRITORES: MATERIAIS DE ENSINO; INFUSÕES INTRAVENOSAS; CATETERISMO PERIFÉRICO; EDUCAÇÃO MÉDICA; EDUCAÇÃO EM ENFERMAGEM.

\begin{abstract}
Aims: To describe a simple and low cost model of peripheral venous access, developed by the Trauma League of Pontifícia Universidade Católica do Rio Grande do Sul, which aims acquiring new skills by the health student/ professional.

Methods: In order to simulate the peripheral access via, we used a rubber tourniquet, which ended in a blind end, simulating a vein, inserted on a latex glove full of fill material. The other extremity was connected to a bag of parenteral solution containing $500 \mathrm{ml}$ of physiologic serum with dye, to simulate blood, through infusion equipos. The bag was hanged higher than the glove, making the gravity laws work. With the flow meter completely open, a pressure is created by the presence of air with liquid inside the infusion equipos and the tourniquet. The first puncture with the catheter takes out the air inside the cannulas, which allows the procedure to be done properly.

Results: The puncture, when done properly, causes the artificial blood to reflow into the puncture catheter, which does not happen when the rubber tourniquet is transfixed. This simulates a real life situation of venous puncture, because the hydrostatic pressure inside the tourniquet is analogous to that found in a vein.

Conclusions: The description of the didactic model allows its reproducibility in an easy way, since the required materials are cheap and easily available. The model provides the initial familiarity with the procedure, making it easier to practice in the patient or in more sophisticated models, indispensable for the improvement of the technique.
\end{abstract}

KEYWORDS: TEACHING MATERIALS; INFUSIONS, INTRAVENOUS; CATHETERIZATION, PERIPHERAL; EDUCATION, MEDICAL; EDUCATION, NURSING. 


\section{INTRODUÇÃO}

A punção de veia periférica é um procedimento comumente realizado no ambiente médico. Suas indicações vão desde o paciente estável que precisa coletar amostras de sangue ou receber soro fisiológico, até o politraumatizado que precisa repor volume ou receber medicações através de uma via rápida de infusão. Tecnicamente simples, o acesso venoso periférico pode ser realizado tanto em ambiente hospitalar quanto em atendimento pré-hospitalar. O processo, porém, não está livre de complicações, principalmente quando é realizado com imperícia. A flebite é uma causa comum de dor e desconforto no paciente que recebe terapia por infusão por meio de cateteres periféricos. Entre 27 e $70 \%$ dos pacientes que recebem esse tipo de terapia desenvolvem algum estágio de flebite. Os hematomas, por outro lado, costumam aparecer quando há transfixação da veia durante uma tentativa de punção sem sucesso. No caso de trauma endotelial na parede venosa, pode haver formação de coágulo e desenvolvimento de trombose. As infecções locais e bacteremias também são complicações frequentes nesse tipo de procedimento, principalmente quando não há realização adequada de técnicas assépticas durante a preparação dos dispositivos e higienização correta do profissional. Sabe-se que mais de $50 \%$ de todas as bacteremias hospitalares epidêmicas relatadas na literatura, entre 1965 e 1991, foram derivadas de algum tipo de acesso vascular. ${ }^{1-4}$

Outra preocupação é com os acidentes ocupacionais envolvendo o profissional da saúde. Lesões perfurantes por agulhas são responsáveis por $80 \%$ de todas as exposições acidentais a sangue, $20 \%$ antes ou durante o uso da agulha e mais de $70 \%$ depois do uso e antes do descarte. ${ }^{5}$ Entre os principais patógenos veiculados pelo sangue estão o HIV, os vírus das hepatites $\mathrm{B}$ e $\mathrm{C}$ e o citomegalovírus. ${ }^{6}$ Assim, o conhecimento e o aperfeiçoamento da técnica de punção e acesso venoso tornam-se essenciais para os profissionais da saúde.

Existem no mercado diversos modelos para desenvolvimento de habilidades em acesso venoso periférico. Esses, em geral, reproduzem meticulosamente a anatomia humana, são seguros, fáceis de manusear, e com excelente capacidade de gerar aprendizado. Por outro lado, eles são de difícil acesso a muitos estudantes e profissionais da saúde, seja pelo seu custo, seja pela sua disponibilidade. Com isso, surgiu a necessidade de desenvolver modelos mais baratos, porém ainda práticos do ponto de vista do treinamento. $\mathrm{Na}$ literatura revisada encontramos um modelo, também desenvolvido por estudantes, semelhante ao modelo que será descrito neste estudo. ${ }^{7} \mathrm{O}$ modelo de acesso venoso que construímos e iremos descrever é simples, de baixo custo e tem o intuito de proporcionar uma experiência inicial da prática de punção e acesso venoso periférico.

\section{MÉTODOS}

Para reproduzir a via periférica de acesso foi utilizado um garrote de borracha, simulando uma veia, com uma extremidade em fundo cego, inserida em uma luva de látex preenchida com estopa de algodão. A outra extremidade do garrote foi conectada a uma bolsa de solução parenteral por equipos de infusão. $\mathrm{Na}$ bolsa de $500 \mathrm{ml}$ de soro fisiológico foi acrescentado corante vermelho, através do injetor, para reproduzir o sangue. A bolsa é instalada em um suporte mais alto que a luva, facilitando a ação da gravidade (Figura 1). Com o regulador de fluxo completamente aberto, cria-se uma pressão através da presença do ar com o líquido dentro dos equipos de infusão e do garrote. A primeira punção com o cateter serve para retirar o ar de dentro das cânulas, já que se trata de um sistema fechado. A partir de então se pode iniciar a prática do procedimento.

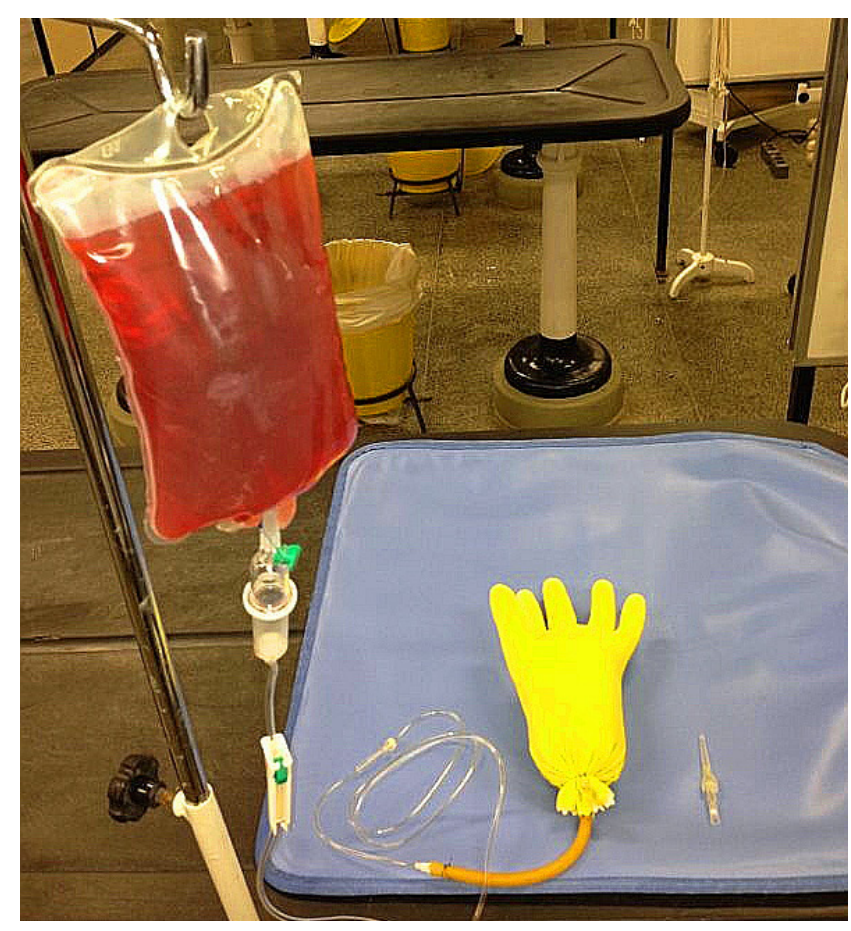

Figura 1. A bolsa e os equipos de infusão devem estar posicionados de maneira a facilitar a ação da gravidade.

Apesar de ser uma simulação, vale ressaltar que a lavagem rigorosa das mãos, indispensável antes da inserção de um cateter vascular periférico e seu 
posterior manuseio, precisa sempre preceder o procedimento. As luvas não substituem a lavagem das mãos e não propiciam proteção completa ${ }^{8} \mathrm{~A}$ adoção de técnicas de higiene e assepsia já devem ser preconizadas no treinamento com o modelo didático, para posteriormente, na prática com o paciente, estarem claras ao profissional.

Quanto ao tipo de cateter periférico usado, vários modelos estão disponíveis comercialmente; cateteres agulhados/scalp (os butterfly), cateteres sobre agulha (os abocath), cateteres sob agulha, cateteres de linha média e cateteres duplo-lúmen. ${ }^{3}$ Para o experimento foi utilizado um cateter do tipo abocath, por ser corriqueiramente usado, principalmente em terapias intravenosas de longa duração.

Este estudo não envolveu diretamente nem indiretamente seres humanos na qualidade de sujeitos de pesquisa. Após consulta ao Comitê de Ética em Pesquisa da Pontifícia Universidade Católica do Rio Grande do Sul, o artigo foi aprovado sem necessidade de cumprir todos os trâmites dos estudos com seres humanos.

\section{RESULTADOS}

Para uma punção eficaz foi necessário estar atento à técnica. $\mathrm{O}$ cateter deve ser inserido em um ângulo de 30 a $45^{\circ}$, paralelamente à "veia", enquanto a "pele" abaixo do local de inserção deve ser tracionada. Devese avançar através do "subcutâneo" até o bisel da agulha entrar na veia obliquamente e ocorrer retorno do "sangue", para então diminuir o ângulo de inserção e progredir dentro da veia. Avança-se o cateter dentro do lúmen da veia, enquanto a agulha é retirada. Podese então conectar o adaptador do equipo de infusão na conexão do cateter, fixá-los e realizar o curativo, onde deve constar a data e o horário do procedimento, o tipo e o comprimento do cateter e as iniciais do profissional. $^{3}$

A punção realizada de forma adequada tem como desfecho o refluxo do sangue artificial por dentro do cateter, ao contrário do que acontece quando não há punção correta ou quando há transfixação do garrote de borracha. Isso simula uma situação real, já que existe uma pressão hidrostática no garrote análoga à encontrada em uma veia. Entretanto, inicialmente algumas dificuldades foram encontradas em relação à saída do sangue artificial pelo cateter, mesmo realizando-se a técnica de punção corretamente. Isso ocorreu quando não foi feita a retirada do ar de maneira adequada de dentro das cânulas. É necessário que esse processo seja feito logo após a conexão da bolsa de solução parenteral com os equipos de infusão e liberação do regulador de fluxo. Caso contrário, formam-se colunas de ar dentro das cânulas, que não conseguem ser empurradas apenas com a força da gravidade.

Após esses resultados satisfatórios, foram desenvolvidos mais cinco modelos idênticos, com intuito de utilizá-los em outra oportunidade. A única diferença é que eles não foram conectados à bolsa de solução parenteral, justamente para preservar suas funcionalidades (Figura 2). Dessa forma, os modelos podem ser armazenados e utilizados posteriormente.

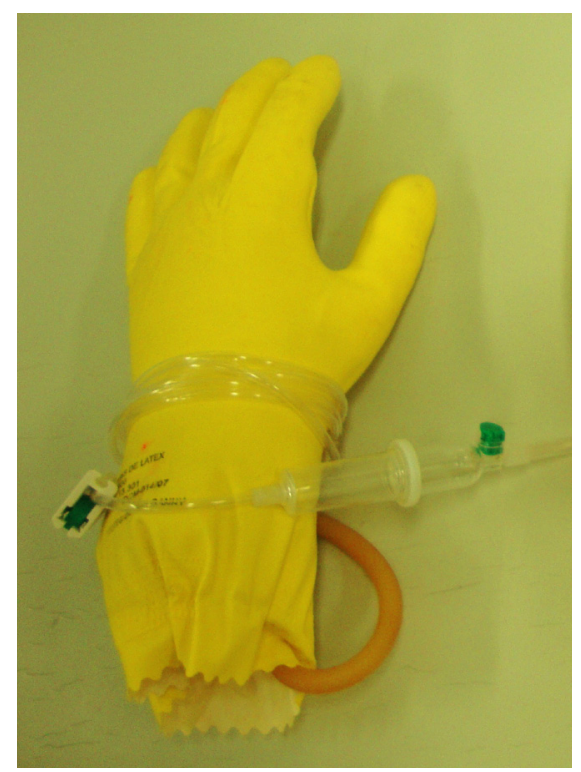

Figura 2. Modelos são guardados pré-prontos (sem a bolsa de solução parenteral).

\section{DISCUSSÃO}

Várias veias podem ser usadas para obter acesso venoso periférico e infundir fluidos, mas as veias das mãos e dos braços são as mais comumente utilizadas. Vários fatores devem ser considerados, tais como facilidade de inserção e acesso, tipo de agulha ou cateter que pode ser empregado, conforto e segurança do paciente. ${ }^{3}$ Nesse modelo experimental não houve preocupação com a reprodução exata da anatomia das veias do local, já que o objetivo foi a prática da técnica de punção venosa de forma simples. Ainda assim, devese lembrar que a região representada pelo modelo é território de algumas veias corriqueiramente utilizadas na terapia intravenosa, como as veias basílica e cefálica do antebraço e as veias metacarpais dorsais. ${ }^{9}$

Práticas relacionadas à terapia intravenosa são empregadas na maioria dos pacientes hospitalizados. ${ }^{3}$ Apesar de ser um procedimento tecnicamente simples, ele está sujeito a diversas complicações, e o profissional 
da saúde deve estar atento a isso. O modelo com a luva mostra-se útil para o aprendizado não só da técnica de punção e acesso venoso periférico, mas também dos procedimentos que precedem e sucedem essa intervenção. As técnicas de punção venosa realizadas de forma asséptica propiciam uma terapia parenteral segura. Por isso, para realização da punção venosa de maneira simulada, devem-se seguir os mesmos passos utilizados na abordagem de um paciente. Isso inclui lavar as mãos, calçar as luvas e preparar o local.

As possibilidades de aprendizado com o modelo prático de punção e acesso venoso podem ser extrapoladas, adequando-as ao público que estará desenvolvendo habilidades com ele. A descrição desse modelo permite sua reprodução de maneira fácil, pois os materiais usados na confecção são de fácil acesso e o método empregado na montagem é simples. O modelo é oportuno para a aquisição de habilidades práticas em terapia endovenosa muito mais pela sua praticidade do que pela sofisticação ou fidedignidade anatômica encontradas em outros modelos. Deve-se ressaltar, entretanto, que, o modelo com a luva serve como instrumento prático inicial. Para o aprimoramento da técnica, é fundamental a prática no paciente ou em modelos mais sofisticados.

\section{REFERÊNCIAS}

1. Comitê do Trauma do Colégio Americano de Cirurgiões. Suporte avançado de vida no trauma para médicos: manual do curso de alunos. $8^{\mathrm{a}}$ ed. Chicago: American College of Surgeons; 2008.

2. Maki DG, Ringer M. Risk factors for infusion-related phlebitis with small peripheral venous catheters. Ann Intern Med. 1991 May 15;114(10):845-54.

3. Phillips LD. Manual de terapia intravenosa. $2^{\mathrm{a}}$ ed. Porto Alegre: Artmed; 2001.

4. Maki DG. The epidemiology and prevention of nosocomial bloodstream infection. In: Program and Abstracts of Third International Conference on Nosocomial Infection. Atlanta: Centers for Disease Control. The National Foundation for Infectious Diseases and American Society for Microbiology; 1990.

5. Jagger J, Hunt EH, Brand-Elnaggar J, Pearson RD. Rates of needle-stick injury caused by various devices in a university hospital. N Engl J Med. 1988 Aug 4;319(5):284-8.

6. Hibberd PL. Patients, needles and health care workers. J Intraven Nurs. 1995 Mar-Apr;18(2):65-76.

7. Miglioransa MH, Laporte GA, Pereira E, Crespo AR. Modelo experimental para treinamento de acesso venoso periférico. In: XV Salão de Iniciação Científica. Porto Alegre: UFRGS; 2003.p. 24-8.

8. Crow S. Asepsis--an indispensable part of the patient's care plan. Crit Care Nurs Q. 1989 Mar;11(4):11-6.

9. Netter FH. Atlas de anatomia humana. $3^{\mathrm{a}}$ ed. Porto Alegre: Artmed; 2003. 